\title{
Viscosity Influence on Flutter Boundary and Limit Cycle Oscillation in Transonic Regime*
}

\author{
Sung-Yeoul LEE** \\ **CADLAB, Mechanical Design Department, Hanyang University \\ Engineering Centre A-518, Hanyang University, Haengdang-dong, Sungdong-gu, Seoul 133-791 Korea \\ E-mail:sungyi09@hanyang.ac.kr
}

\begin{abstract}
Viscosity has a great influence on nonlinearity especially in the transonic regime: however only preliminary research into the viscous effect on the aeroelstic response was conducted. Nonlinearity of the flowfield assumed to be the main source of flutter behaviours. This paper reports the results of intensive numerical simulations carried out on 2D aerofoil sections to investigate the effect of viscosity on flutter boundary and especially on limit cycle oscillation (LCO) characteristics. Unsteady Euler and Navier-Stokes flow solvers are developed for use in aeroelastic analysis with the moving grid method, while the aeroelastic response is calculated with the aeroelastic equations of motion in two degrees of freedom. The flow solvers are verified with simulations of pitching aerofoil. Two aeroelastic test cases ranging from subsonic to transonic flow regimes $\left(\mathrm{M}_{\infty}=0.3 \sim 0.92, \mathrm{Re}_{\mathrm{c}}=12.3 \times 10^{6}\right)$ were simulated in depth to obtain flutter boundaries as well as characteristics of LCO. The results indicate that the LCO occurs in the subsonic flow regime as well as in the transonic flow regime with far greater intensity caused by viscous effect. It has been shown that the weak divergence is found to be a transonic aeroelastic phenomenon.
\end{abstract}

Key words: Aeroelasticity, Limit Cycle Oscillation, Flutter Boundary, Viscous Effects

\section{Introduction}

Aircraft structures, being extremely flexible, are prone to distortion under loads caused by aerodynamic forces. These aerodynamic forces are dependent on the geometry of the structure. Distortion in the geometry of the structure due to the aerodynamic forces produces a new state of aerodynamic forces, and consequently a new state of distorted geometry. This sequence of interactions between the aerodynamic forces and the elastic forces in the structure is known as aeroelasticity. Two distinct type of aeroelastic problems occur. First, the interaction may exhibit divergent tendencies in a too flexible structure, leading to failure. In this type of problem static or steady state systems of aerodynamic and elastic forces produce such aeroelastic phenomena as divergence and control reversal. Secondly, the interaction involves the inertia of the structure as well as aerodynamic and elastic forces. Dynamic aerodynamic loads may induce oscillations of structure components. This problem produces such aeroelastic phenomena as flutter, buffeting and dynamic response.

In flutter analysis, two noticeable phenomena can be observed. The first one is the transonic dip of the flutter speed. This is a drop in the flutter speed in the transonic flow region and is believed to be due to the massive flow separation. The second one is a limit cycle oscillation (LCO). It is the instability in the fluid-structure interactions, which evolves into a sustained periodic oscillation with limited amplitude. A series of researchers $\left(\right.$ Edwards $^{(8)}$, Bendiksen ${ }^{(5)}$ ) believe that the development of nonlinearity in the transonic flow regime due to shock and separation is the main source of the LCO behaviour. This 
nonlinearity of the flow is the dominant feature of transonic flow. This is the reason why they assumed the appearance of LCO in transonic flutter.

The most effective way of analyzing flutter in the transonic regime to capture the nonlinearity is via a time-marching method ${ }^{(1)(5)(8)(10)(11)(12)(14)(18)(19)(21)(22)}$. The aeroelastic system is simulated in time for various fluid-structure systems. The response is plotted in time to see if it is diverging or converging, or if it converges to a LCO. Numerical studies of flutter in transonic flow have been mostly performed with the perturbation theory, Euler and Navier-Stokes equations. Isogai ${ }^{(11)}$ developed a TSP (transonic small perturbation) code, which can be used for the entire transonic Mach number range (from sub-critical Mach number to Mach number above one). It predicted the sharp and deep transonic dip of the flutter boundary. Kousen \& Bendiksen ${ }^{(12)}$ studied the effects of shock dynamics on flutter in transonic flow by integrating the explicit Euler fluid solver with the structure solver without considering the structural damping. Nonlinear aeroelastic phenomena such as LCO, weak divergence and transonic dip were reported. In the process, large amplitude LCOs in the response were observed. Kousen \& Bendiksen ${ }^{(12)}$ discussed the transonic flutter behaviour as an energy exchange between fluid and structure. Few Navier-Stokes solvers are applied for the fluid model in 2D aeroelastic applications. In the early work of Alonso et al. ${ }^{(2)}$, the Navier-Stokes study was limited to verification of the flow solver as a potential tool in aeroelastic applications.

Renewed interest in LCO in the transonic regime led to a series of experimental (Schewe et al. ${ }^{(18)}$, Dietz et al. ${ }^{(7)}$ ) and numerical research papers (Weber et al. ${ }^{(22)}$, Tang et al. ${ }^{(19)}$ ) on supercritical aerofoil NLR 7301. However their research was limited to transonic cases with focus on the LCOs and their amplitudes. In contrast, there are more published works (Guruswamy ${ }^{(10)}$ ) for 3-D configurations. These have typically analyzed flexible wings over a limited range of flow conditions without investigation of LCO. The study of Lee-Rausch \& Batina ${ }^{(14)}$ was carried out for a 3-D test case of the AGARD (Advisory Group for Aeronautical Research and Development) Wing 445.6 following investigation of their prior work with Euler solver. They found that the use of Navier-Stokes solver improved the flutter boundary in the transonic and supersonic regimes. However, even with Navier-Stokes solver, the calculated flutter boundary is still higher compared to the results from the experiment in the supersonic region, and these were restricted to finding the flutter boundary.

It is known that the shock wave and especially the phase lag between shock motion and the aerofoil pitching and plunging motions play the dominant role in the transonic dip mechanism and the LCO phenomenon. The importance of the role of shock wave is also pointed out by Ashley ${ }^{(3)}$. This highlights its importance in the prediction of transonic flow with respect to the LCO in the transonic regime, illustrating the need for the Navier-Stokes investigation to account viscous effect on the aeroelastic studies.

This paper presents the extensive numerical aeroelastic simulations of the Euler and the Navier-Stokes flow solvers for subsonic and transonic flow regimes of $\mathrm{M}_{\infty}=0.3 \sim 0.92$ at $\mathrm{Re}_{\mathrm{c}}=12.3 \times 10^{6}$ to investigate the effects of viscosity on aeroelastic stability. Simulations represent the small oscillatory input for short time, such as gust, on the symmetrical aerofoil section of straight and swept back wing configurations. The finite volume flow solvers are used with the moving grid method, while the aeroelastic response is calculated with the aeroelastic equations of motion in two degrees of freedom. The transonic dip in the flutter boundary and LCO behaviours are investigated in detail with the aeroelastic test cases covering subsonic to transonic.

\section{Nomenclature}

$a_{h}:$ dimensionless offset between the elastic axis and the mid-chord

$b$ : semi-chord length 


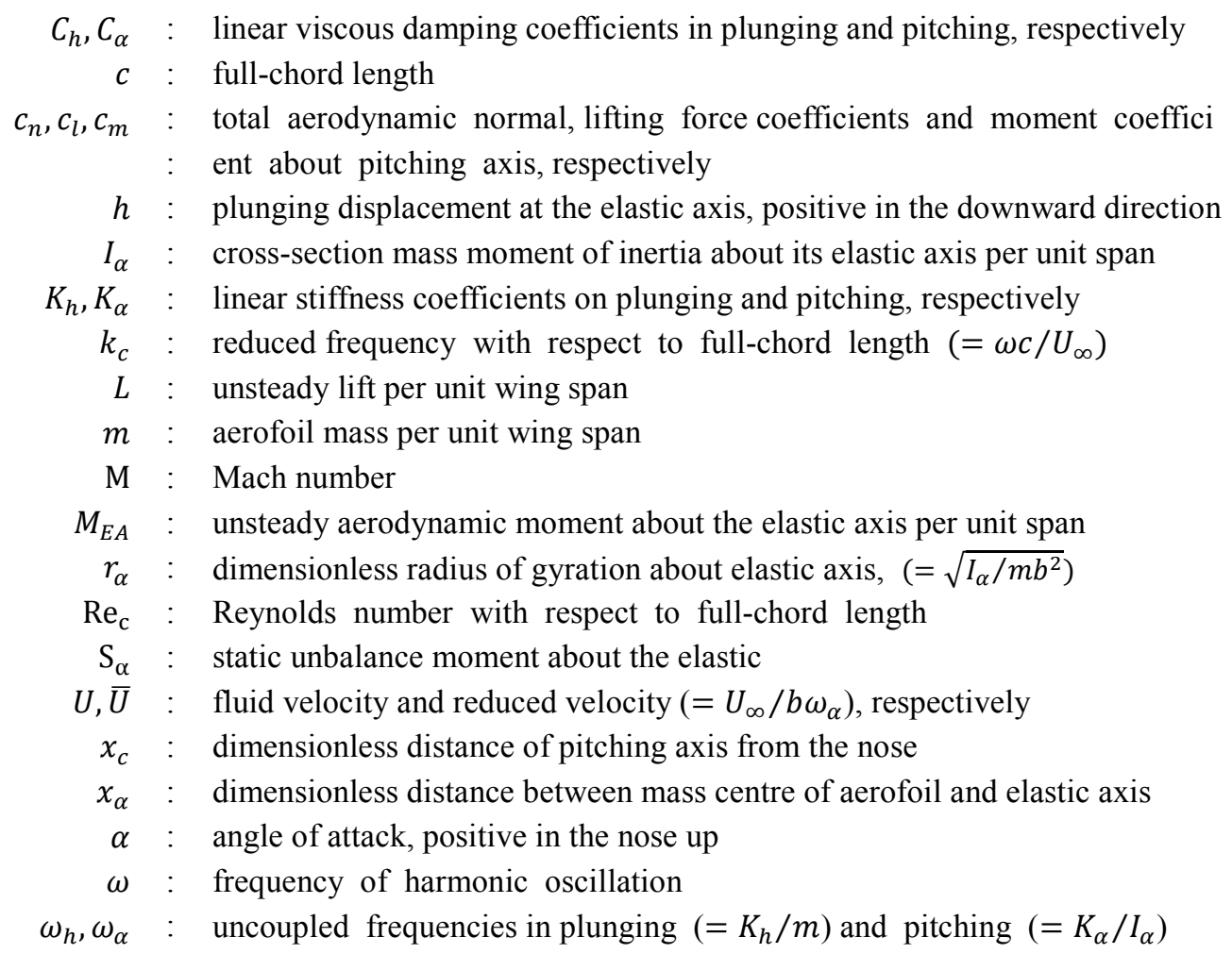

Flutter index : $\bar{U}_{F} / b \omega_{\alpha} \sqrt{\mu}$

Subscripts

$\infty \quad$ : free stream

\section{Aeroelastic Solver}

The flow analysis and the structural response are coupled in the time marching process. The instantaneous values of forces from the unsteady flow solution are used in a simultaneous solution of the structural solution. Since an implicit system needs to be solved iteratively at each time step for the flow equations, the structure equations can be coupled fully with the flow equation.

\subsection{Aerodynamics solver}

The physical problem under consideration is that of compressible viscous air flow involving shock waves, shear layers (including boundary layers) and their interactions. The mathematical model used to represent the problem is the two-dimensional compressible thin-layer Navier-Stokes equation.

The law of conservation of mass, momentum and energy over an area $S$ bounded by a contour line $l$ can be expressed in integral form as

$$
\frac{\partial}{\partial t} \int_{S} \mathbf{q} d S+\int_{l}(H \cdot \mathbf{n}) d l=0
$$

where $\mathbf{q}$ is the vector of conserved variables $(\rho, \rho u, \rho v, \rho E)^{T}$, in which $\rho$ is the density of the fluid, $u$ and $v$ are the components of fluid velocity in a Cartesian co-ordinate system and $E$ is the energy of the fluid particle element, $E=e+0.5 \times \rho\left(u^{2}+v^{2}\right)$. In the above equation, $\mathbf{n}$ is the outward pointing unit vector normal to the line $l$.

The flux tensor $H$ is made up from contributions that may be conveniently grouped together as convection and diffusion terms, denoted by the superscripts $i$ and $v$ respectively. In a Cartesian co-ordinate system, the convective flux and the corresponding components of the diffusive flux may be written as 


$$
\begin{aligned}
& H_{x}^{i}=\left[\begin{array}{c}
\rho u_{c} \\
\rho u u_{c}+p \\
\rho v u_{c} \\
u_{c}(\rho E+p)+u_{g} p
\end{array}\right], H_{y}^{i}=\left[\begin{array}{c}
\rho v_{c} \\
\rho u v_{c} \\
\rho v v_{c}+p \\
v_{c}(\rho E+p)+v_{g} p
\end{array}\right] \text { and } \\
& H_{x}^{v}=\left[\begin{array}{c}
0 \\
\tau_{x x} \\
\tau_{x y} \\
u \tau_{x x}+v \tau_{x y}-q_{x}
\end{array}\right], H_{y}^{v}=\left[\begin{array}{c}
\tau_{x y} \\
\tau_{y y} \\
u \tau_{x y}+v \tau_{y y}-q_{y}
\end{array}\right]
\end{aligned}
$$

in which $\tau$ is the shear stress tensor and $q$ is the heat flux. The thin-layer approximation is used when evaluating $\tau$, i.e. derivatives of flow variables with respect to the generalized co-ordinate in the wall-tangential direction are neglected. The contra variant velocities $u_{c}$ and $v_{c}$ are

$$
u_{c}=u-u_{g} \text { and } v_{c}=v-v_{g}
$$

where $u_{g}$ and $v_{g}$ represent the grid velocities, $d x / d t$ and $d y / d t$, with which the integration boundaries move.

In order to obtain a closed mathematical system, auxiliary relations describing the interdependence of the thermodynamic variables are introduced. For a perfect gas, the gas-law relates the pressure $p$, density and temperature $T$ by $p=\rho R T$, where $R$ is the universal gas constant. The internal energy $e$ is defined by $e=p / \rho(\gamma-1)$, where $\gamma$ is the ratio of specific heats.

The laminar viscosity in the shear stress is determined as a function of $T$ by Sutherland's formula. In turbulent cases, the Reynolds-averaged Navier-Stokes equations are used with the Baldwin-Lomax ${ }^{(4)}$ algebraic turbulence model.

In the present aerodynamic solver, a finite volume spatial discretisation method is used on a structure grid to solve the Navier-Stokes equations. Oshers approximate Riemann solver ${ }^{(16)}$ is used for the spatial discretisation of the inviscid flux terms while central differences are employed for the spatial discretisation of the viscous flux term. The present approach to the spatial discretisation has been demonstrated ${ }^{(17)}$ that both shock waves and shear layers accurately described allowing the reliable prediction of shock waves-boundary layer interactions. Higher order spatial accuracy is achieved using MUSCL (monotonic upstream centred scheme for conservation laws) interpolation ${ }^{(20)}$ while flux limiter is utilised for the removal of the spurious oscillations commonly found in the region of strong discontinuities, for example sock waves.

Implicit methods for the calculation of unsteady flow require the solution of large, sparse system of linear equations, $[\mathbf{A}]\{x\}=\{b\}$. Direct solvers, such as LU decomposition, are inappropriate for problems of this size hence iterative method of GMRES (generalised minimal residual) is used to solve these linear equations in the present approach. The system matrix $[\mathbf{A}]$ is generally ill-conditioned for this class of problems which has severe consequences for the convergence behaviour of GMRES (and most alternative iterative solver).

In order to improve the condition of the system matrix, and hence the convergence behaviour of the iterative solver, pre-conditioning technique is required. Approximate factorisation (AF) scheme is introduced as this method provides a fast and efficient means of preconditioning the unsteady Navier-Stokes equations.

\subsection{Structural solver}

Structural modelling of the two degrees of freedom aeroelastic configuration for a rigid aerofoil can be described with a spring, mass and damper system which simulates the banding and twisting of a wing. The equations governing the aeroelastic structural dynamics system and the numerical method used are given below. 
The aeroelastic equations of motion for two degrees of freedom can be written as

$$
\begin{aligned}
m \ddot{h}+S_{\alpha} \ddot{\alpha}+C_{h} \dot{h}+K_{h} h & =-L \\
S_{\alpha} \ddot{h}+I_{\alpha} \ddot{\alpha}+C_{\alpha} \dot{\alpha}+K_{\alpha} \alpha & =M_{E A}
\end{aligned}
$$

The matrix equation for the response analysis in the non-dimensional form is

$$
[\mathbf{M}]\left\{\begin{array}{c}
\xi^{\prime \prime} \\
\alpha^{\prime \prime}
\end{array}\right\}+[\mathbf{C}]\left\{\begin{array}{c}
\xi^{\prime} \\
\alpha^{\prime}
\end{array}\right\}+[\mathbf{K}]\left\{\begin{array}{c}
\xi \\
\alpha
\end{array}\right\}=\frac{4}{\pi \mu k_{c}^{2}}\left\{\begin{array}{c}
-c_{l} \\
2 c_{m}
\end{array}\right\}
$$

where the mass, damping and spring stiffness matrices are given by

$$
[\mathbf{M}]=\left[\begin{array}{cc}
1 & x_{\alpha} \\
x_{\alpha} & r_{\alpha}^{2}
\end{array}\right],[\mathbf{C}]=\left[\begin{array}{cc}
S_{h} & 0 \\
0 & \varsigma_{\alpha}
\end{array}\right],[\mathbf{K}]=\left(\frac{2}{\bar{U} k_{c}}\right)^{2}\left[\begin{array}{cc}
\left(\frac{\omega_{h}}{\omega_{\alpha}}\right)^{2} & 0 \\
0 & r_{\alpha}^{2}
\end{array}\right]
$$

In the present structural solver, the direct integration method based on linear variation in acceleration is employed to find the time-history dynamic responses in the aeroelastic system of Eq. (3). The major advantage of this scheme lies in its simplicity that only one time step and one numerical calculation are required. The velocities and displacement of a small time interval $\Delta t$ can be expressed as

$$
\begin{aligned}
& \{\dot{q}\}_{t+\Delta t}=\{\dot{q}\}_{t}+\frac{\Delta t}{2}\{\ddot{q}\}_{t}+\frac{\Delta t}{2}\{\ddot{q}\}_{t+\Delta t} \\
& \{q\}_{t+\Delta t}=\{q\}_{t}+\Delta t\{\dot{q}\}_{t}+\frac{\Delta t}{2}\{\ddot{q}\}_{t}+\frac{\Delta t}{2}\{\ddot{q}\}_{t+\Delta t}
\end{aligned}
$$

Substituting into Eq. (3) gives

$$
\{\ddot{q}\}_{t+\Delta t}=[\mathbf{F}]\left[\{p\}_{t+\Delta t}-[\mathbf{C}]\{v\}_{t}-[\mathbf{K}]\{w\}_{t}\right]
$$

where $\{p\}_{t+\Delta t}$ are the aerodynamic loads obtained from the aerodynamic solver,

$$
[\mathbf{F}]=\left[[\mathbf{M}]+\frac{\Delta t}{2}[\mathbf{C}]+\frac{\Delta t^{2}}{6}[\mathbf{K}]\right]^{-1},\{v\}_{t}=\{\dot{q}\}_{t}+\frac{\Delta t}{2}\{\ddot{q}\}_{t},\{w\}_{t}=\{q\}_{t}+\Delta t\{\dot{q}\}_{t}+\frac{\Delta t^{2}}{3}\{\ddot{q}\}_{t}
$$

Thus, the integrated value of next time level $t+\Delta t$ is obtained. The matrix $[\mathbf{F}]$ needs only to be formed once since it is independent of time. The scheme is a step-by-step time integration finite difference approach. This method is conditionally stable, however, the time step required to produce accurate results for a structural response solution was found to be smaller than the maximum time step needed for the flow solver. Therefore, in the present study the same time step was used for both aerodynamic and structural response computations.

\section{Numerical Simulations of Pitching Aerofoil}

As the LCO exhibits the rapidly oscillating movement, the flow solver developed requires ability to describe the unsteady flow of pitching aerofoil. Therefore, investigation of the unsteady flow around a pitching aerofoil has been carried out to confirm the validity of the present numerical computation to accurately resolve unsteady flow and its associated phenomena such as shock-boundary interactions. The cases of an aerofoil pitching about the quarter chord point were examined with the instantaneous angle of attack in the following form,

$$
\begin{array}{ll}
\alpha(\tau)=\alpha_{m}+\alpha_{0} \sin \left(k t^{*}\right) & \text { for NACA0012 } \\
\alpha(\tau)=\alpha_{m}+\alpha_{0} \cos \left(k t^{*}\right) & \text { for NACA64A010 }
\end{array}
$$

where $\alpha_{m}, k=\omega c / U_{\infty}$ and $\alpha_{0}$ are the mean angle of attack, frequency and amplitude of the motion respectively. Motions of pitching aerofoil are encountered in the study of aeroelasticity, thus the ability to predict flow fields of this type is a prerequisite for a successful prediction of the LCO and the flutter boundary.

The test cases were selected for two important flow regimes. First, with a high subsonic freestream velocity and large pitch amplitude, this flow regime exhibits the leading edge separation and the formation of a strong dynamic stall vortex. The subsequent behaviour of 
this vortex dominates the development of the unsteady flowfield and the resultant forces and moments. These flow characteristics have a great influence on the divergence behaviour of flutter. Second, with a transonic freestream velocity, this flow regime is dominated by the effect of an unsteady shock system and its interaction with a boundary layer. This regime has great importance in understanding the LCO and the transonic dip in flutter boundary prediction.

The unsteady aerodynamic measurements of AGARD aeroelastic configurations have been collected in the report by Lambourne ${ }^{(13)}$. From his report, two oscillatory experimental data sets were used to evaluate the flow regimes mentioned above. The experimental conditions are summarized in Table 1.

Table 1. Pitching aerofoil experimental conditions

\begin{tabular}{cccccccc}
\hline Case & Aerofoil & $\mathbf{M}_{\infty}$ & $\mathbf{R e}_{\mathbf{c}} \times \mathbf{1 0}^{\mathbf{6}}$ & $\boldsymbol{\alpha}_{\boldsymbol{m}}$ & $\boldsymbol{\alpha}_{\mathbf{0}}$ & $\boldsymbol{k}$ & $\boldsymbol{x}_{\boldsymbol{c}}$ \\
\hline CT2 & NACA 0012 & 0.6 & 4.8 & 3.16 & 4.59 & 0.1622 & 0.25 \\
\hline CT6 & NACA 64A010 & 0.796 & 12.56 & 0 & 1.02 & 0.4040 & 0.248 \\
\hline
\end{tabular}

Figure 1 presents the comparison of the present computed force and moment with experimental measurements for the case of CT2. The comparison shows close agreement in the lower incidence range but deteriorates as the aerofoil pitches up to the maximum incidence. The normal force coefficient, $c_{n}$ is in line with that of experimental data, but appears to be under-predicted over the latter stages of the upstroke of the aerofoil. These discrepancies are more apparent in the pitching moment coefficients over a similar incidence range. The computations are in good overall agreement with the experimental data and the shape of the curve on the lift and moment is well captured.
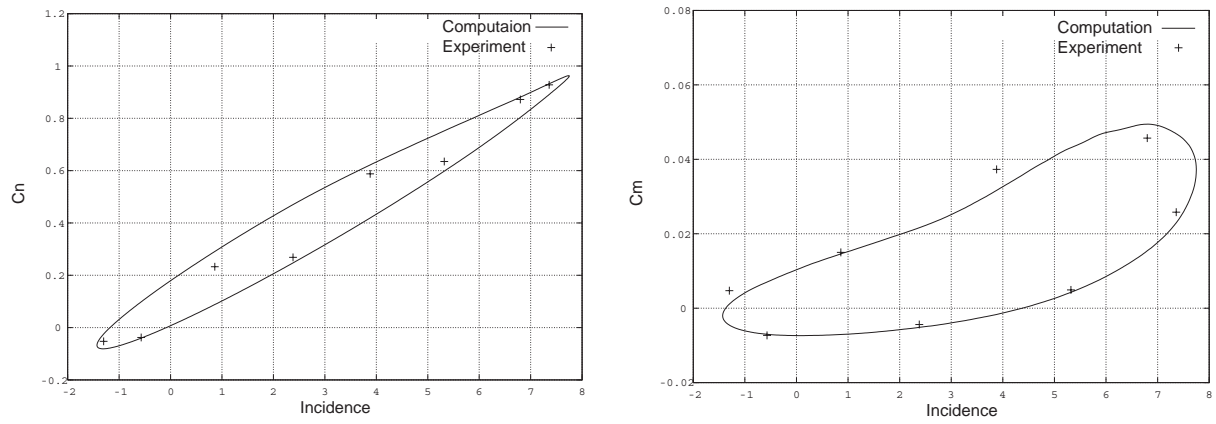

Fig.1 Comparison of computed and experiment force and moment (CT2).

In the test case CT6, the freestream Mach number is close to transonic flow ensuring that shock waves are present over the full range of incidences. The aerofoil used in this test case has been subjected to the aeroelastic response in present study. Hence, both Euler and Navier-Stokes solvers are tested for the effects of viscosity as in the aeroelastic study. The present computed lift and pitching moment coefficients are compared with experimentally measured values in Fig. 2. The lift coefficient comparison shows good agreement for both
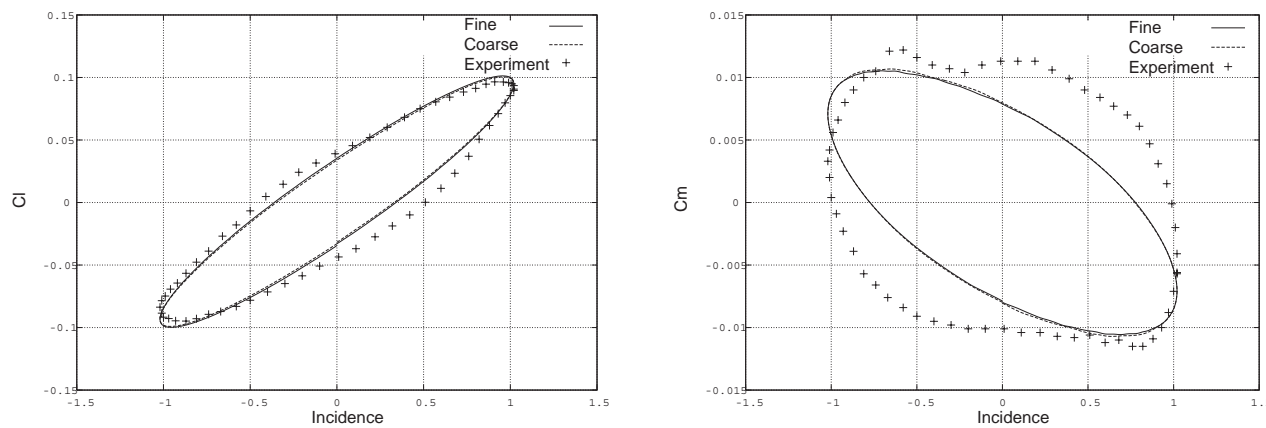

Fig. 2 Comparison of computed and experiment force and moment (CT6). 
Euler and Navier-Stokes solvers while the Euler solver exhibits slightly more over prediction than the Navier-Stokes. However, the pitching moment comparison is unsatisfactory for both solvers and again the Euler solver produces a greater over prediction than the Navier-Stokes. The same disagreement was obtained by Venkatakrishnan ${ }^{(21)}$ and Gaitonde \& Fiddes ${ }^{(9)}$, and they suggested that this could be the result of incorrect integration of the experimental pressure distributions.

The present approach for solving unsteady problems on an oscillating aerofoil is validated for use in aeroelasticity. The numerical results have generally shown good agreement when compared with experimental measurements for a pitching aerofoil over a range of flow conditions. From the results, it is clear that the turbulence modelling (algebraic Baldwin-Lomax ${ }^{(4)}$ ) is capable of providing a good flow development of attached flow. It suffers in the flow development following boundary layer separation. This is attributed largely to weakness in the turbulence modelling approaches including other high order models. Another point to be addressed is that the present calculations are performed with the turbulence model applied over the full chord of the aerofoil. However, in the experiment, there will be regions of laminar and transitional flow in the proximity of the aerofoil leading edge. The extent of this region is unknown and one might assume that this will have an important effect on the flow development especially, when the shock wave and separation point are close to the transition region.

\section{Numerical Simulations of Aeroelastic Response}

Transonic aeroelastic response solutions are presented for two test cases to investigate the effects of nonlinearity in the transonic flow regime. Numerical simulations of these configurations were to verify the viscous effects on flutter boundary prediction and to investigate the viscous effects on the flutter by use of the Navier-Stokes flow solver. The aeroelastic parameter values for the test cases are tabulated in Table 2.

Table 2. Aeroelastic test cases for rigid aerofoil configurations.

\begin{tabular}{cccc}
\hline \multicolumn{2}{c}{ Test case } & I & II \\
\hline & Aerofoil & NACA64A006 & NACA64A010 \\
\cline { 2 - 4 } & $a_{h}$ & -0.2 & -2.0 \\
\cline { 2 - 4 } Aeroelastic & $x_{\alpha}$ & 0.2 & 1.8 \\
\cline { 2 - 4 } parameters & $r_{\alpha}^{2}$ & 0.5385 & 1.86548 \\
\cline { 2 - 4 } & $\omega_{h} / \omega_{\alpha}$ & 0.34335 & 1.0 \\
\cline { 2 - 4 } & $\mu$ & 10.0 & 60.0 \\
\hline
\end{tabular}

Test case I simulates the vibrational characteristics of a typical chordwise section of an unswept wing and test case II simulates that of a sweptback wing. Test case I and II were originally studied by using Euler equations (Kousen \& Bendiksen ${ }^{(12)}$ ) and the transonic small perturbation equations (Isogai ${ }^{(11)}$ ) respectively. A system with two degrees of freedom was investigated by both authors and used in present study as base configurations. The forced amplitude of $1^{\circ}$ and the forced oscillation frequency of $k=1.0$ were fixed for all cases in the present study as used in the previous study (Alonso \& Jameson ${ }^{(1)}$ ). All the calculation was initiated by forcing the system to oscillate, usually 3-4 cycles, according to the mode as a given reference (Alonso \& Jameson ${ }^{(1)}$ ). Then the aerofoil was left free in order to obtain the aeroelastic response.

The Hopf bifurcation (Marsden \& McCracken $^{(15)}$ ) is used to model dynamic instabilities, including flutter. The reduced velocity, $\bar{U}=U_{\infty} / b \omega_{\alpha}$ is generally used as the bifurcation parameter, hence the speed index, $\bar{U}_{F} / b \omega_{\alpha} \sqrt{\mu}$ where $\bar{U}_{F}$ is the reduced velocity at the flutter speed, based on the reduced velocity is used for the flutter boundary 
parameter as practiced in the aeroelasticity community. Most calculations were computed with both Euler and Navier-Stokes equations to verify the viscous effects on the flutter boundary prediction, especially for the transonic dip phenomenon. The LCO and weak divergence in the transonic flow regime phenomena were investigated.

\subsection{Test case I}

NACA 64 A006 aerofoil was studied by Kousen \& Bendiksen ${ }^{(12)}$ with an Euler solver. The choice of this case for them and present study lies on its convenience from a computational standpoint that the resulting flutter frequency is relatively high, which allows the aeroelstic solver to track many cycles of oscillations in a given number of iterations.

Present numerical simulations showed LCO and divergence behaviours, and Kousen \& Bendiksen $^{(12)}$ concluded from their study that limit cycle behaviour appears to be a transonic phenomenon, wherein shocks on the surfaces of the aerofoil interact dynamically with the aerofoil motion to force the system into a limit cycle. They have also observed a weak divergence motion of the system to a nonzero static equilibrium position. This equilibrium position does not correspond to the one predicted by static analyses. Unfortunately their investigation does not cover the transonic dip phenomenon. This test case provides preliminary investigation of the effects of viscosity on the flutter boundary prediction and LCO phenomenon.

The selected aeroelastic responses are shown in Fig. 3 to illustrate that LCO is not only for a transonic phenomenon as Kousen $\&$ Bendiksen ${ }^{(12)}$ suggested. The phenomenon occurs in the whole flow regime with different strengths and ranges. The main source of LCO would be the formation of vortex flow in the subsonic region and the nonlinearity in the flow in the transonic region. The observation of responses suggests that a region of LCO is much greater as the flow reaches the transonic regime, and reduces as the flow approaches the sonic condition. Hence the LCO can be treated as a phenomenon which is important in transonic flow aeroelastic characteristics. The amplitude of LCO ranged up to the angle of attack at 13 degree, when the flow pattern would be dominated by the shock induced separation. These high amplitudes would result from excluding the structural damping terms in the structural solver.
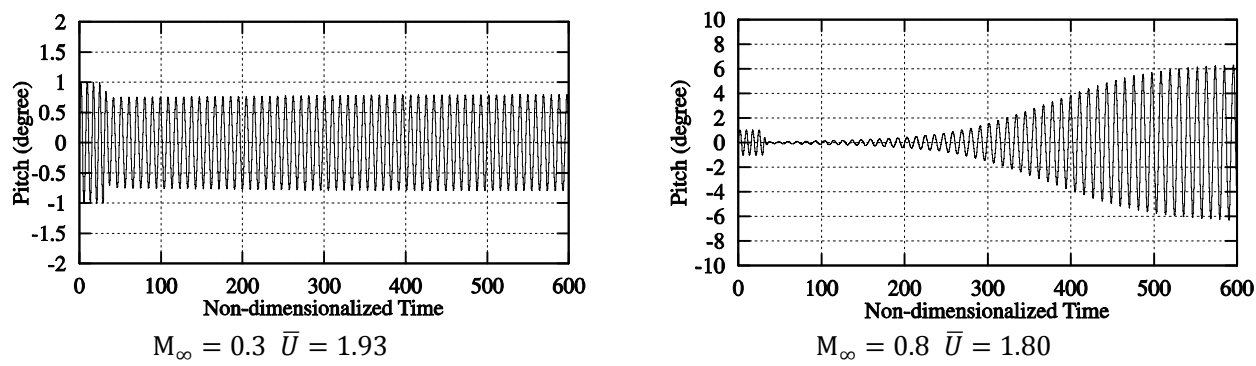

Fig. 3 Aeroelastic responses at LCO (test case I).

Three phenomena are of interest when examining the responses. First, the amplitude of the LCO increased with increasing $\bar{U}$ and Mach number. Second, the higher $\bar{U}$ LCO showed significant flattening, indicating the presence of higher harmonics in the response. Finally, the growth to the final LCO was more rapid at a higher value of $\bar{U}$. The three basic trends occurred in all the transonic Mach number cases studied. The identical trends are demonstrated in the plunging oscillations as well. The frequency spectrum analysis shows the exact peak frequency for both pitching and plunging oscillations.

The flutter boundary determined by present Navier-Stokes solver is compared with previous results by Kousen \& Bendiksen ${ }^{(12)}$ in Fig. 4. They performed a numerical test with Euler solver at Mach numbers of 0.25, 0.60, 0.80, 0.85, 0.87 and 0.92. However they did not provide any data regarding the flutter velocity for Mach numbers when limit cycle 
behaviour did not occur. The data presented are for Mach number above 0.80 where the limit cycle occurred. The flutter point is obtained and shown in Figure 4. The transonic dip was clearly shown for present Navier-Stokes solutions. The comparison of flutter point on Mach numbers of $\mathrm{M}_{\infty}=0.8,0.87$ and 0.92 shows reasonable agreement. However there is not sufficient information to discuss the transonic dip phenomenon with this case. Therefore, this will be left to Case II. This test case serves as a good preliminary test for present study.

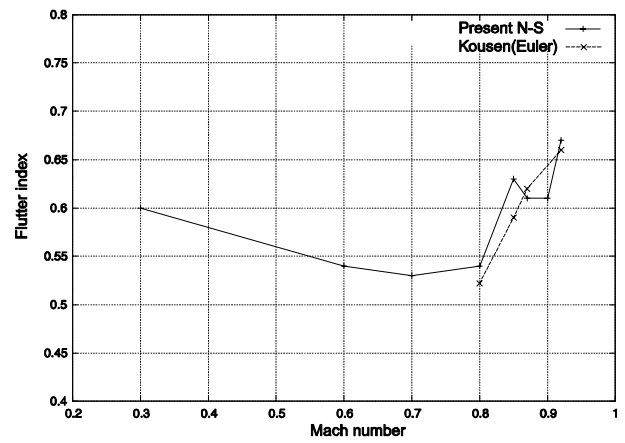

Fig. 4 Flutter boundary for test case I (NACA 64A006).

\subsection{Test case II}

NACA 64A010 aerofoil is simulated for the vibrational characteristics of a swept wing. This test case is a follow up of test case A by Isogai ${ }^{(11)}$ and has been chosen by other researches $^{(1)(6)(8)}$ and the present study for comparison purposes since it covers the flutter points from the subsonic to sonic flow regime.

From the present numerical simulations, the growth of the LCO and the divergence bifurcation is observed. Kousen \& Bendiksen ${ }^{(12)}$ also showed a weak divergence on a symmetric aerofoil in the test case I configurations. This is a phenomenon where the mean incidence approaches a nonzero value as time goes to infinity. It differs from a classical divergence condition where the aerofoil twists until failure. This phenomenon of weak divergence is investigated in this test case.

As shown in Fig. 5, the bifurcation divergence is produced in both Navier-Stokes and Euler solvers with a wider range in the Navier-Stokes solver. The converged nonzero value in the Navier-Stokes solver increases with increasing value of $\bar{U}$ from $1.6^{\circ}$ at $\bar{U}=10.0$ to $2.5^{\circ}$ at $\bar{U}=13.0$. Interestingly the converged nonzero value changes from a positive angle of attack to negative at $\bar{U}=15.0$. This behaviour would be the result of a phase shift between the aeroelastic structural system and the unsteady aerodynamics. The absolute value of the converged angle of attack increases with increasing values of $\bar{U}$. However a significant difference between the phenomena described by Kousen \& Bendiksen ${ }^{(12)}$, and in the one observed here is that the former appears below the bifurcation point while the latter appears above the region of the LCO. Examination of aeroelastic responses from two solvers indicates that a wide region of LCO occurs with the Navier-Stokes solver. The LCO occurs at the reduced velocity from $\bar{U}=2.0$ to $\bar{U}=9.0$ with the Navier-Stokes solver, showing far greater and lower range than with the Euler solver. This lower LCO observation with the Navier-Stokes solver shows that the viscosity has a great influence on
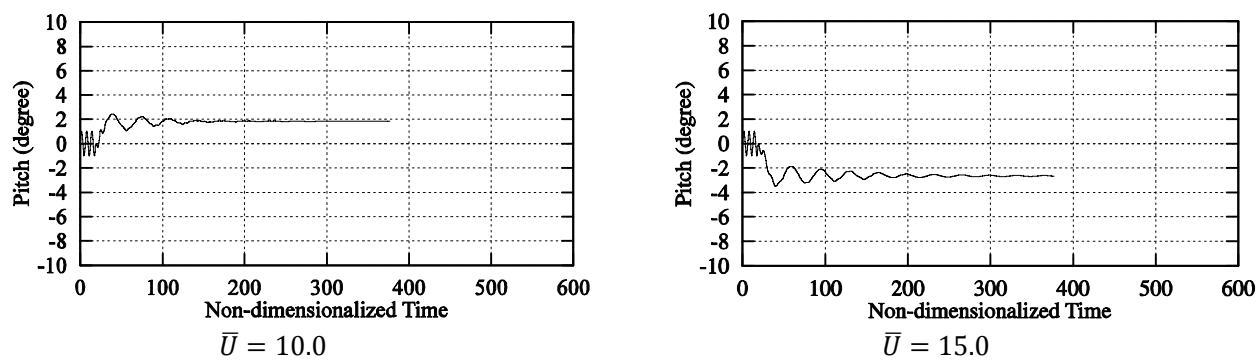

Fig. 5 Divergence bifurcation on test case II at $\mathrm{M}_{\infty}=0.875$. 
the onset and the region of LCO in the transonic regime. Increase in $\bar{U}$ from 10.0 to 15.0 resulted in a weak divergence, i.e., nonzero values of mean incidence and plunge. Further increases in the value of $\bar{U}$ resulted in a recurrence of LCO with a high amplitude at zero incidence and plunge.

The flowfields at small angles of attack during aeroelastic response are presented in Fig. 6 to highlight the viscous effects. Flowfields are obtained during rapidly oscillating aeroelastic response therefore it was not possible to obtain the same angle of attack for Euler and Navier-Stokes flow solvers for comparison. From the closest angle of attack obtained for both solvers, it can be seen that the positions of shock waves and separations of flow are completely different between Euler and Navier-Stokes solvers. The Euler solver was not able to capture the movement of the shock waves and the formation of separated flow at these small angles of attack on a rapidly oscillating aerofoil. The oscillatory frequency for the presented flowfields is higher than the forced oscillation frequency of 1.0 which is already much higher than that of the experimental pitching aerofoil. The phase lag is an important feature of LCO and one can imagine from the flowfields that the Navier-Stokes solver provides better phase lag interaction than the Euler solver. Comparison of the positions of shock waves and separations highlights the role of viscosity on the onset of LCO again.

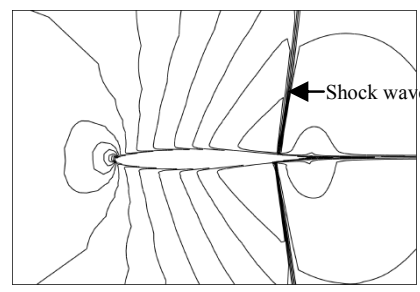

Euler $\alpha=-0.79^{\circ}$

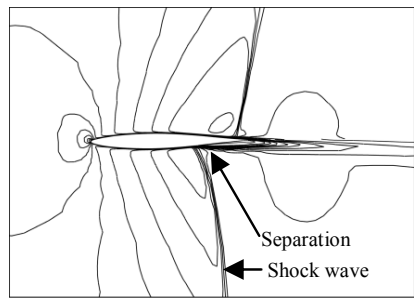

$\mathrm{N}-\mathrm{S} \alpha=-0.75^{\circ}$

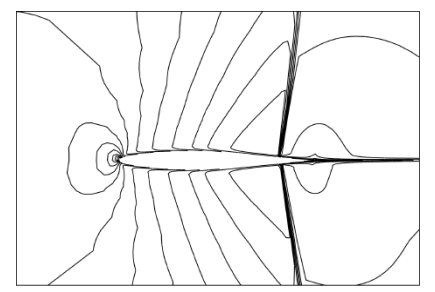

Euler $\alpha=0.08^{\circ}$

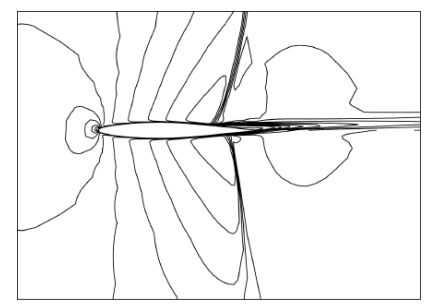

$\mathrm{N}-\mathrm{S} \alpha=0.02^{\circ}$

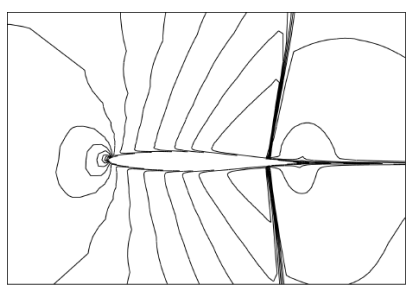

Euler $\alpha=0.81^{\circ}$

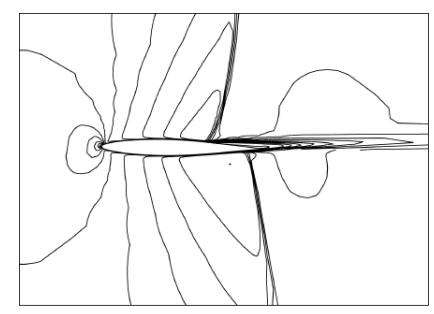

$\mathrm{N}-\mathrm{S} \alpha=0.82^{\circ}$

Fig. 6 Positions of shock waves and separations between Euler and Navier-Stokes during aeroelastic response $\left(\mathrm{M}_{\infty}=0.875\right)$.

The flutter boundary is presented in Fig. 7 for comparison with previous results by TSP and Euler solvers. The transonic dip is well predicted by all of the results. The bottom of the dip is close to $\mathrm{M}_{\infty}=0.85$ and all the results deviate less than $10 \%$ from this value except the Navier-Stokes solution. However, the discrepancy occurs in the Mach number range $0.875<\mathrm{M}_{\infty}<1.0$. Multiple flutter points are observed at a range of Mach numbers in the neighbourhood of $\mathrm{M}_{\infty}=0.85$ in the results of Alonso \& Jameson ${ }^{(1)}$ and Edwards ${ }^{(8)}$ while similar behaviour is observed in the neighbourhood of $\mathrm{M}_{\infty}=0.9$ in the results of Bendiksen $\&$ Kousen ${ }^{(6)}$ and the present Euler solver. The disagreement in the Euler results in this range can only be explained by the different spatial meshes used. C type mesh is used in Bendiksen \& Kousen $^{(6)}$ and present study while an O type mesh is used by Alonso \& Jameson $^{(1)}$. These multiple flutter points might be tried since the TSP and Euler solvers did not provide any results in the Mach number region of $0.85<\mathrm{M}_{\infty}<0.9$. The flutter points over $\mathbf{M}_{\infty}=0.9$ are only obtained with a TSP solver while the Euler solver and the Navier-Stokes 
solver did not detect any flutter points in this region. This would be the result of numerical accuracy of the solver. A significant difference is observed at $\mathrm{M}_{\infty}=0.9$ between Bendiksen $\&$ Kousen $^{(6)}$ and the present Euler. This discrepancy is possibly due to the high mesh density in the present study. The Navier-Stokes solution shows a much lower flutter boundary in the transonic regime. These multiple flutter points are not observed in the Navier-Stokes solution and there are no flutter points over $M_{\infty}=0.9$. Therefore it is believed that the multiple flutter points and the flutter points over $\mathrm{M}_{\infty}=0.9$ observed in the TSP or Euler solvers are due to inadequate description of the phase-lag of shockwave motion. The importance of viscous effects on the flutter boundary is discovered in this test case with the viscosity encouraging the onset of LCO at lower values.
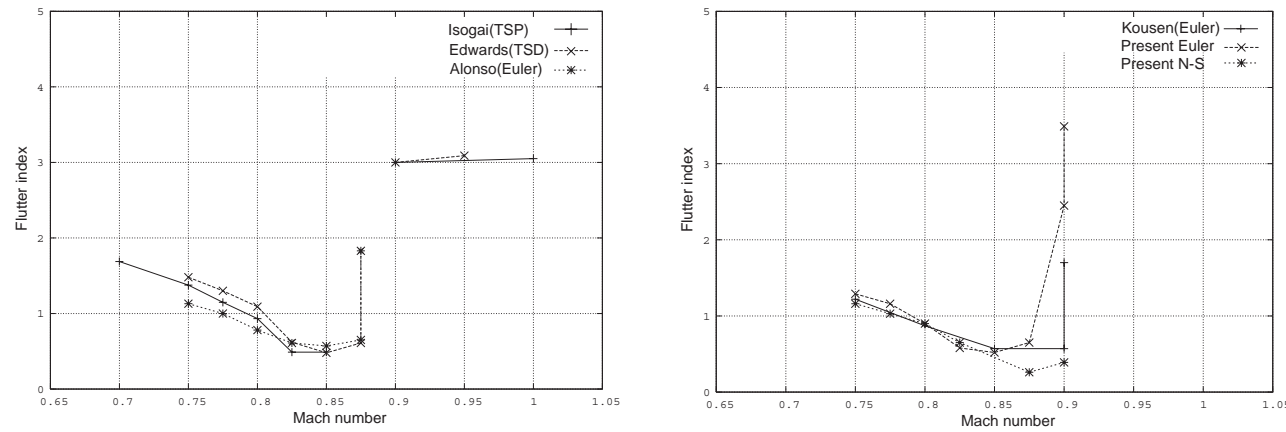

Fig. 7 Comparison of flutter boundaries for test case II (NACA 64A010).

\section{Conclusions}

In order to capture the effect of nonlinearity of flow on the flutter boundary and on the behaviour of aeroelastic response, the unsteady Euler and Navier-Stokes solvers are developed for use in the aeroelastic analysis of a two degree of freedom system. The Reynolds-averaged Navier-Stokes equations together with an algebraic turbulence model were employed for the unsteady flow solver to account for the viscous effects. To obtain the detailed aeroelastic time response of the aerofoil motion, the time domain approach was employed for analysis rather than the frequency domain approach.

The aeroelastic response is dependent on phase-lag between the motion of the structure and the unsteady aerodynamics. In turn, the phase-lag is dominated with nonlinearity of flow produced by vortex formation in subsonic flow regime and by shock-structure interactions in transonic flow regime. Therefore, the precise location of shocks and their associated flow characteristics are important in the shock-structure interactions in transonic flow. Inclusion of the viscous effects enables better flowfield description associated with the shock-boundary layer interactions resulting in improved unsteady aerodynamic coefficients. This led to more accurate aeroelastic response calculations.

From the results, it should be noted that the LCO phenomenon seems to be the phenomenon dominated by nonlinearity of the flowfield especially in the transonic flow regime and greatly influenced by the viscous effect of flow. The onset and region of LCO are lowered and widened respectively as a result of viscosity. This indicates the importance of shock dynamics on the aeroelastic response in the transonic flow regime. The divergence bifurcation only occurred in the transonic regime indicating the possibility that this is a transonic flow regime phenomenon. The present study helps a better understanding of the importance of viscosity of flow in the flutter prediction.

\section{References}

(1)Alonso, J.J. and Jameson, A., "Fully-implicit time-marching aeroelastic solutions",AIAA Paper-94-0056, 1994. 
(2)Alonso, J, Martinelli, L. and Jameson, A., "Multigrid unsteady Navier-Stokes calculations with aeroelastic applications", AIAA Paper 95-0048, 1995.

(3)Ashley, H., "Role of shocks in the sub-transonic flutter phenomenon", J. Aricraft, Vol.17, pp187-197, 1980.

(4)Baldwin, B.S. and Lomax, H., "Thin-layer approximation and algebraic model for separated turbulent flow", AIAA Paper 78-257,1978.

(5)Bendiksen, O.O., "A New Approach to Computational Aeroelasticity", AIAA 32nd Structures, Structural Dynamics, and Material Conference, AIAA, pp 1712-1727 (AIAA 91-0930), 1991.

(6)Bendiksen, O.O. and Kousen, K.A., "Transonic flutter analysis using the Euler equations", AIAA Paper 87-0911-CP, Monterey, California, 1987.

(7)Dietz, G., Schewe, G., Mai, H., "Experiments on heave/pitch limit-cycle oscillations of a supercritical airfoil close to the transonic dip", Journal of Fluids and Structures 19, pp 1-16, 2004.

(8)Edwards, J.W., "Time marching transonic flutter solutions including Angle of Attack effects", Journal of Aircraft, 1983, vol. 20, pp 899-906.

(9)Gaitonde, A.L. and Fiddes, S.P., “A moving mesh system for the calculation of unsteady flows", AIAA Paper 93-0461, 1993.

(10)Guruswamy, G.P., "Navier-Stokes computations on swept-tapered wings, including flexibility", AIAA Paper 90-1152, 1990.

(11)Isogai, K., "Numerical study of transonic flutter of a two-dimensional airfoil", NAL TR-617T, National Aerospace Laboratory, July 1980.

(12)Kousen, K.A. and Bendiksen, O.O., "Non-linear aspects of the transonic stability problem", AIAA Paper-88-2306, April 1988.

(13)Lambourne, N.C., "Compendium of unsteady aerodynamic measurements", AGARD Report 702, 1982.

(14)Lee-Rausch, E.M. and Batina, J.T., "Wing flutter computations using an aerodynamic based on the Navier-Stokes equations", Journal of Aircraft, vol. 33, no. 6, pp 1139-1148, 1996.

(15)Marsden, J.E. and McCracken, M., "The Hopf bifurcation and its applications", Applied Mathematical Sciences, 19, 1976.

(16)Osher, S., Solomon, F., "Upwind difference schemes for hyperbolic systems of conservation laws", Mathematics of computation V.38 No. 158, 1982.

(17)Qin, N., Soriba, K. W., Richard, B.E. "Shock-Shock, Shock-Vortex interactions and aerodynamic heating in hypersonic corner flow", Aeronautical Journal V. 95, pp 152-160 1992.

(18)Schewe, G., Mai, H., Dietz, G., "Nonlinear effects in transonic flutter with emphasis on manifestations of limit cycle oscillations", Journal of Fluids and Structures 18, pp 3-22, 2003.

(19)Tang, L., Bartels, R.E., Chen, P.C., Liu, D.D., "Numerical investigation of transonic limit cycle oscillations of a two-dimensional supercritical wing", Journal of Fluids and Structures 17, pp 29-41, 2003.

(20)Van Leer, B., "A second order sequel to Gudonovs method" Journal of computational physics V. 32, pp 101-136.

(21)Venkatakrishnan, V., "Computation of Unsteady Transonic Flows over Moving Airfoils", PhD Thesis, Department of Mechanical and Aerospace Engineering, Princeton University, U.S.A., 1986.

(22)Weber, S., Jones, K.D., Ekaterinaris, J.A., Platzer, M.F., “Transonic flutter computations for the NLR 7301 supercritical airfoil”, Aerospace Science and Technology 5, pp 293-304, 2001 . 